\title{
Correlation Functions in Super Liouville Theory
}

\author{
E. Abdalla, (1) M. C. B. Abdalla, ${ }^{(2)}$ D. Dalmazi, (2) ${ }^{\text {and Koji Harada }}{ }^{(1),(a)}$ \\ (1) Instituto de Física, Universidade de São Paulo, CP 20516, São Paulo, Brazil \\ (2) Instituto de Física Teórica, UNESP, Rua Pamplona 145, CEP 01405, São Paulo, Brazil
}

(Received 27 August 1991)

\begin{abstract}
We calculate three- and four-point functions in super Liouville theory coupled to a super Coulomb gas on world sheets with spherical topology. We first integrate over the zero mode and assume that a parameter takes an integer value. We find the amplitudes, give plausibility arguments in favor of the result, and formally continue the parameter to an arbitrary real number. Remarkably the result is completely parallel to the bosonic case.
\end{abstract}

PACS numbers: $11.17 .+\mathrm{y}, 04.60 .+\mathrm{n}, 11.30 . \mathrm{Pb}$

The matrix model definition of $2 \mathrm{D}$ gravity is proving to be very powerful in calculating correlation functions [1], although it seems difficult to generalize the results to supersymmetric theories. On the other hand, in the continuum approach (Liouville theory) [2-5] it is difficult to calculate correlation functions, while its supersymmetric generalization (super Liouville theory) [6-8] is well known. Recently, however, several authors [9-13] have exactly calculated correlation functions in the continuum approach to conformal matter fields coupled to 2D gravity (see also Ref. [14]). They have used a technique based on the integration over the Liouville zero mode, and their results agree with those obtained earlier in the discrete approach (matrix models). It is thus very urgent to extend the continuum method [15] to the supersymmetric case, i.e., superconformal matter fields coupled to 2D supergravity.

The aim of this Letter is to calculate the three- and four-point functions in super Liouville theory coupled to superconformal matter with the central charge $\hat{c}<1$, represented as a super Coulomb gas [16]. Our approach is close to that of Di Francesco and Kutasov [10]. The result is remarkable and is very parallel to the bosonic case; it amounts to a redefinition of the cosmological constant and of the primary superfields, resulting in the same amplitudes as those of the bosonic theory. The strategy is to first calculate the amplitudes in terms of a conformally invariant integral. We find the result of the integral assuming analyticity (see also [17]), using its symmetries and asymptotic behavior. We give plausibility arguments in favor of the result.

The relevant framework has been given by Distler, Hlousek, and Kawai [8]. With a translation invariant measure, the total action is given by $S=S_{\mathrm{SL}}+S_{M}$,

$$
\begin{aligned}
& S_{\mathrm{SL}}=\frac{1}{4 \pi} \int d^{2} z \hat{E}\left(\frac{1}{2} \hat{D}_{\alpha} \Phi_{\mathrm{SL}} \hat{D}^{\alpha} \Phi_{\mathrm{SL}}\right. \\
&\left.-Q \hat{Y} \Phi_{\mathrm{SL}}-4 i \mu e^{\alpha+\Phi_{\mathrm{SL}}}\right), \\
& S_{M}=\frac{1}{4 \pi} \int d^{2} z \hat{E}\left(\frac{1}{2} \hat{D}_{\alpha} \Phi_{M} \hat{D}^{\alpha} \Phi_{M}+2 i \alpha_{0} \hat{Y} \Phi_{M}\right),
\end{aligned}
$$

where $\Phi_{\mathrm{SL}}$ and $\boldsymbol{\Phi}_{M}$ are super Liouville and matter superfields, respectively (see Refs. $[8,18]$ ). The matter sector has the central charge $\hat{c}_{m}=1-8 \alpha_{0}^{2}$. The parameters $Q$ and $\alpha \pm$ are given by

$$
\begin{aligned}
& Q=2\left(1+\alpha_{0}^{2}\right)^{1 / 2}, \\
& \alpha_{ \pm}=-\frac{1}{2} Q \pm \frac{1}{2}\left(Q^{2}-4\right)^{1 / 2}=-\frac{1}{2} Q \pm\left|\alpha_{0}\right| .
\end{aligned}
$$

The (gravitationally dressed) primary superfield $\tilde{\Psi}_{\mathrm{NS}}$ is given by

$$
\begin{aligned}
& \tilde{\Psi}_{\mathrm{NS}}(\mathbf{z}, k)=d^{2} z \hat{E} e^{i k \Phi_{M}(\mathbf{z})} e^{\beta(k) \Phi_{\mathrm{SL}}(\mathbf{z})}, \\
& \beta(k)=-\frac{1}{2} Q+\left|k-\alpha_{0}\right| .
\end{aligned}
$$

Note that the expressions for $\alpha_{ \pm}$and $\beta(k)$ are the same in terms of $Q$ and $\alpha_{0}$ as those of the bosonic theory.

Screening charges in the matter sector are of the form $d^{2} z e^{i d_{ \pm} \Phi_{M}(\mathrm{z})}$, where $d_{ \pm}$are the two solutions of $\frac{1}{2} d\left(d-2 \alpha_{0}\right)=\frac{1}{2}$. In this Letter, however, we will concentrate on the case without screening charges. The case with screening charges, $N$-point $(N \geq 4)$ functions, and the inclusion of the Ramond sector will be discussed elsewhere.

We shall calculate three-point functions of the primary field $\tilde{\Psi}_{\text {NS }}$ on world sheets with spherical topology (without screening charges), that is,

$$
\left\langle\prod_{i=1}^{3} \int \tilde{\Psi}_{\mathrm{NS}}\left(\mathrm{z}_{i}, k_{i}\right)\right\rangle \equiv \int\left[\mathscr{D}_{\hat{E}} \Phi_{\mathrm{SL}}\right]\left[\mathscr{D}_{\hat{E}} \Phi_{M}\right] \prod_{i=1}^{3} \tilde{\Psi}_{\mathrm{NS}}\left(\mathrm{z}_{i}, k_{i}\right) e^{-s} \text {. }
$$

Our first step is to integrate over the zero modes,

$$
\begin{aligned}
& \left\langle\prod_{i=1}^{3} \int \tilde{\Psi}_{\mathrm{NS}}\left(\mathbf{z}_{i}, k_{i}\right)\right\rangle \equiv 2 \pi \delta\left(\sum_{i=1}^{3} k_{i}-2 \alpha_{0}\right) \mathcal{A}\left(k_{1}, k_{2}, k_{3}\right), \\
& \left.\mathcal{A}\left(k_{1}, k_{2}, k_{3}\right)=\Gamma(-s)\left(\frac{-\pi}{2}\right)^{3}\left(\frac{i \mu}{\pi}\right)^{s}\left\langle\int \prod_{i=1}^{3} d^{2} \tilde{z}_{i} e^{i k_{i} \Phi_{M}\left(\tilde{z}_{i}\right)} e^{\beta_{i} \Phi_{\mathrm{SL}}\left(\tilde{z}_{i}\right)} \iint d^{2} z e^{\alpha_{+} \Phi_{\mathrm{SL}}(\mathrm{z})}\right)^{s}\right\rangle_{0},
\end{aligned}
$$


where $\langle\cdots\rangle_{0}$ denotes the expectation value evaluated in the free theory $(\mu=0)$ and we have absorbed the factor $\left[\alpha_{+}(-\pi / 2)^{3}\right]^{-1}$ into the normalization of the path integral. The parameter $s$ is defined as

$$
s=-\frac{1}{\alpha_{+}}\left(Q+\sum_{i=1}^{3} \beta_{i}\right)
$$

In general, $s$ can take any real value and there is no obvious way of calculating the path integral. However, if we assume that $s$ is a non-negative integer [8-13], this is just a free-field correlator. Under this assumption, we evaluate the path integral, and formally continue $s$ to noninteger values. For $s$ as a non-negative integer,

$$
\begin{aligned}
\mathcal{A}\left(k_{1}, k_{2}, k_{3}\right) & =\Gamma(-s)\left(\frac{-\pi}{2}\right)^{3}\left(\frac{i \mu}{\pi}\right)^{s} \int \prod_{i=1}^{3} d^{2} \tilde{z}_{i} \prod_{i=1}^{s} d^{2} z_{i} \prod_{i<j}^{3}\left|\tilde{\mathbf{z}}_{i j}\right|^{2 k_{i} k_{j}-2 \beta_{i} \beta_{j}} \prod_{i=1}^{3} \prod_{j=1}^{s}\left|\tilde{z}_{i}-z_{j}-\tilde{\theta}_{i} \theta_{j}\right|^{-2 \alpha_{i} \beta_{i}} \prod_{i<j}^{s}\left|\mathbf{z}_{i j}\right|^{-2 \alpha^{2}} \\
& =\Gamma(-s)\left(\frac{-\pi}{2}\right)^{3}\left(\frac{i \mu}{\pi}\right)^{s} \int \prod_{i=1}^{s} d^{2} z_{i} d^{2} \tilde{\theta} \prod_{i=1}^{s}\left|z_{i}+\tilde{\theta} \theta_{i}\right|^{-2 \alpha_{+} \beta_{1}} \prod_{i=1}^{s}\left|1-z_{i}\right|^{-2 \alpha_{+} \beta_{2}} \prod_{i<j}^{s}\left|\mathbf{z}_{i j}\right|^{-2 \alpha_{+}^{2}} .
\end{aligned}
$$

We have divided by the $\widehat{\mathrm{SL}_{2}}$ volume by setting $\tilde{z}_{1}=0, \tilde{z}_{2}=1, \tilde{z}_{3}=\infty, \tilde{\theta}_{2}=\tilde{\theta}_{3}=0$, and $\tilde{\theta}_{1} \equiv \tilde{\theta}$. The integral is a supersymmetric generalization of formula (B.9) of Ref. [19]. Alternatively, using $\Phi_{\mathrm{SL}}=\phi+\theta \psi+\bar{\theta} \bar{\psi}$, we can write

$$
\begin{aligned}
& \mathcal{A}\left(k_{1}, k_{2}, k_{3}\right)=\Gamma(-s)\left(\frac{-\pi}{2}\right)^{3}\left(\frac{i \alpha^{2}+\mu}{\pi}\right)^{s} \beta_{\uparrow}^{2} \int \prod_{i=1}^{s} d^{2} z_{i} \prod_{i=1}^{s}\left|z_{i}\right|^{-2 \alpha_{+} \beta_{1}}\left|1-z_{i}\right|^{-2 \alpha_{+} \beta_{2}} \\
& \times \prod_{i<j}^{s}\left|z_{i}-z_{j}\right|^{-2 \alpha_{+}^{2}}\left\langle\bar{\psi} \psi(0) \bar{\psi} \psi\left(z_{1}\right) \cdots \bar{\psi} \psi\left(z_{s}\right)\right\rangle_{0} .
\end{aligned}
$$

This is nonvanishing only for $s$ odd; we thus write $s \equiv 2 m+1$. One may evaluate $\langle\bar{\psi} \cdots \bar{\psi}\rangle_{0}$ and $\langle\psi \cdots \psi\rangle_{0}$ independently. Since the rest of the integrand is symmetric, one may write the result in a simple form by relabeling coordinates:

$$
\mathcal{A}\left(k_{1}, k_{2}, k_{3}\right)=-i\left(\frac{-\pi}{2}\right)^{3} \Gamma(-s) \Gamma(s+1) \frac{1}{\alpha_{+}^{2}}\left(\frac{\alpha^{2}+\mu}{\pi}\right)^{s} I^{m}(\alpha, \beta ; \rho),
$$

where

$$
\begin{aligned}
I^{m}(\alpha, \beta ; \rho)=\frac{\alpha^{2}}{2^{m} m !} \int d^{2} w \prod_{i=1}^{m} & d^{2} \zeta_{i} d^{2} \eta_{i}|w|^{2 \alpha-2}|1-w|^{2 \beta} \\
& \times \prod_{i=1}^{m}\left|w-\zeta_{i}\right|^{4 \rho}\left|w-\eta_{i}\right|^{4 \rho}\left|\zeta_{i}-\eta_{i}\right|^{-2} \prod_{i=1}^{m}\left|\zeta_{i}\right|^{2 \alpha}\left|\eta_{i}\right|^{2 \alpha}\left|1-\zeta_{i}\right|^{2 \beta}\left|1-\eta_{i}\right|^{2 \beta} \\
& \times \prod_{i, i}^{m}\left|\zeta_{i}-\eta_{j}\right|^{4 \rho} \prod_{i<j}^{m}\left|\zeta_{i}-\zeta_{j}\right|^{4 \rho}\left|\eta_{i}-\eta_{j}\right|^{4 \rho}
\end{aligned}
$$

and $\alpha=-\alpha+\beta_{1}, \beta=-\alpha+\beta_{2}$, and $\rho=-\frac{1}{2} \alpha_{+}^{2}$.

We now have to calculate $I^{m}(\alpha, \beta ; \rho)$. Using the residual $\operatorname{SL}(2, C)$ transformations it is actually not difficult to show that $I^{\prime \prime \prime}$ has the following symmetries:

$$
I^{m}(\alpha, \beta ; \rho)=I^{m}(\beta, \alpha ; \rho)=I^{m}(-1-\alpha-\beta-m \rho, \beta ; \rho) .
$$

Thus we may write $I^{\prime \prime}(\alpha, \beta ; \rho)$ in the following way:

$$
\begin{aligned}
I^{m}(\alpha, \beta ; \rho)=C_{m}(\alpha, \beta ; \rho) \prod_{i=0}^{m} \Delta & (1+\alpha+2 i \rho) \Delta(1+\beta+2 i \rho) \Delta(-\alpha-\beta+(2 i-4 m) \rho) \\
& \quad \times \prod_{i=1}^{m} \Delta\left(\frac{1}{2}+\alpha+(2 i-1) \rho\right) \Delta\left(\frac{1}{2}+\beta+(2 i-1) \rho\right) \Delta\left(-\frac{1}{2}-\alpha-\beta+(2 i-4 m-1) \rho\right),
\end{aligned}
$$

where $C_{m}(\alpha, \beta ; \rho)$ has the same symmetries as $I^{m}(\alpha, \beta ; \rho)$, and where $\Delta(x) \equiv \Gamma(x) / \Gamma(1-x)$. By looking at the large- $\alpha$ behavior,

$$
I^{m}(\alpha, \beta ; \rho) \sim \alpha^{-2 m-2(2 m+1) \beta-4 \rho m(2 m+1)},
$$

one can confirm that $C_{m}(\alpha, \beta ; \rho)$ is, as a function of $\alpha$, bounded as $|\alpha| \rightarrow \infty$ and assumed analytic. This means that $C_{m}(\alpha, \beta ; \rho)$ is independent of $\alpha$, and by symmetry, of $\beta$ as well; $C_{m}=C_{m}(\rho)$.

It is hard to calculate $C_{m}(\rho)$. For this purpose, it is useful to consider the simpler integral

$$
J^{m}(\alpha, \beta ; \gamma ; \rho)=\int \prod_{i=1}^{m} d^{2} \zeta_{i} d^{2} \eta_{i} \prod_{i=1}^{m}\left|\zeta_{i}\right|^{2 \alpha}\left|\eta_{i}\right|^{2 \alpha}\left|1-\zeta_{i}\right|^{2 \beta}\left|1-\eta_{i}\right|^{2 \beta} \prod_{i, j}^{m}\left|\zeta_{i}-\eta_{j}\right|^{4 \rho} \prod_{i<j}^{m}\left|\zeta_{i}-\zeta_{j}\right|^{4 \rho}\left|\eta_{i}-\eta_{j}\right|^{4 \rho} \prod_{i=1}^{m}\left|\zeta_{i}-\eta_{i}\right|^{4 \gamma} \text {. }
$$

By using similar arguments, one may obtain

$$
J^{m}(\alpha, \beta ; \gamma ; \rho)=\tilde{C}_{m}(\gamma ; \rho) \prod_{i=0}^{m-1} \Delta(1+\alpha+2 i \rho) \Delta(1+\beta+2 i p) \Delta(-1-\alpha-\beta-2 \gamma+(2 i-4 m+2) \rho)
$$




$$
\times \prod_{i=1}^{m} \Delta(1+\alpha+\gamma+(2 i-1) \rho) \Delta(1+\beta+\gamma+(2 i-1) \rho) \Delta(-1-\alpha-\beta-\gamma+(2 i-4 m+2) \rho) .
$$

Again, it is very difficult to calculate $\tilde{C}_{m}(\gamma ; \rho)$. Unfortunately we could not get it in a rigorous way. A series of trials and errors, however, led us to the following form:

$$
\tilde{C}_{m}(\gamma ; \rho)=\frac{\pi^{2 m} m !}{2^{m}}[\Delta(-(\gamma+\rho))]^{2 m} \prod_{i=1}^{m} \Delta(1+2(\gamma+i \rho)) \Delta(1+\gamma+(2 i-1) \rho) .
$$

This is consistent with

$$
\tilde{C}_{1}(\gamma ; \rho)=\frac{1}{2} \pi^{2}[\Delta(-(\gamma+\rho))]^{2} \Delta(1+\gamma+\rho) \Delta(1+2(\gamma+\rho))
$$

and with the two other (calculable) cases $\rho=0$ and $\gamma=0$ (up to symmetry factors). It is very difficult to get anything else consistent with these constraints. And a posteriori it seems to be correct since it gives a physically reasonable result. Let us assume that (18) is correct and see its consequences. The two integrals are related by

$$
I^{m}(\epsilon, \beta ; \rho)=-\left(\pi / 2^{m} m !\right) \Delta(1+\epsilon) \Delta(1+\beta) \Delta(-\epsilon-\beta) J^{m}\left(2 \rho, \beta ;-\frac{1}{2} ; \rho\right) .
$$

Therefore $C_{m}(\rho)=-\left(\pi / 2^{m} m !\right) \tilde{C}_{m}\left(-\frac{1}{2} ; \rho\right) \Delta\left(\frac{1}{2}-\rho\right) \Delta\left(\frac{1}{2}+(2 m+1) \rho\right)$. If we substitute (18) we get

$$
C_{m}(\rho)=-\frac{\pi^{2 m+1}}{2^{2 m}}\left[\Delta\left(\frac{1}{2}-\rho\right)\right]^{2 m+1} \prod_{i=1}^{m} \Delta(2 i \rho) \prod_{i=0}^{m} \Delta\left(\frac{1}{2}+(2 i+1) \rho\right) .
$$

Notice that after Eq. (15) we have assumed that $C_{m}(\alpha, \beta ; \rho)$ is an analytic function of $\alpha$ and $\beta$. Although a full proof of analyticity of $C_{m}(\alpha, \beta ; \rho)$ is outside the scope of the present paper, we give three arguments of plausibility which give a strong basis to the result. First, $C_{m}$ can be derived from $\tilde{C}_{m}\left(\gamma=-\frac{1}{2} ; \rho\right)$ using (20). The proof of the analyticity of the latter relies on the results of Ref. [18]. Thus one can prove analyticity in that particular case. Second, we suppose that nothing new should happen for particular values of $m$ (or $s$ ). At $m=1$, the calculation can be done explicitly. At last, one can compare the pole structure with that of string amplitude in a sphere with punctures (we thank A. Schwimmer for this comment).

Now we are ready to write down the amplitude. Without loss of generality, we can choose $k_{1}, k_{3} \geq \alpha_{0}, k_{2} \leq \alpha_{0}$. By using (5), (8), and $\sum_{i=1}^{3} k_{i}=2 \alpha_{0}$, one gets

$$
\beta= \begin{cases}\rho(1-s) & \left(\alpha_{0}>0\right), \\ -\frac{1}{2}-\rho s & \left(\alpha_{0}<0\right) .\end{cases}
$$

It is easily seen that, for $\alpha_{0}>0, \mathcal{A}=0$ identically, as in the bosonic theory. For $\alpha_{0}<0$, there are many cancellations, leading to

$$
\begin{aligned}
I^{m}(\alpha, \beta ; \rho) & =C_{m}(\rho) \prod_{i=0}^{m} \Delta\left(\frac{1}{2}-(2 i+1) \rho\right) \prod_{i=1}^{m} \Delta(-2 i \rho) \Delta(1+\alpha+2 m \rho) \Delta\left(\frac{1}{2}-\alpha+\rho\right) \\
& =(-1)^{m+1} \frac{\pi^{2 m+1}}{(m !)^{2}}\left[\Delta\left(\frac{1}{2}-\rho\right)\right]^{2 m+1}(4 \rho)^{-2 m} \Delta(1+\alpha+2 m \rho) \Delta\left(\frac{1}{2}-\alpha+\rho\right) .
\end{aligned}
$$

We finally obtain the three-point function:

$$
\begin{aligned}
\mathcal{A}\left(k_{1}, k_{2}, k_{3}\right) & =\left(\frac{-i \pi}{2}\right)^{3}\left[\frac{\mu}{2} \Delta\left(\frac{1}{2}-\rho\right)\right]^{s} \Delta\left(\frac{1}{2}-\frac{s}{2}\right) \Delta(1+\alpha+2 m \rho) \Delta\left(\frac{1}{2}-\alpha+\rho\right) \\
& \left.=\left[\frac{\mu}{2} \Delta\left(\frac{1}{2}-\rho\right)\right]_{j=1}^{s} \prod_{j=1}^{3}-\frac{i \pi}{2}\right) \Delta\left(\frac{1}{2}\left[1+\beta_{j}^{2}-k_{j}^{2}\right]\right) .
\end{aligned}
$$

By redefining the cosmological constant and the primary superfield $\hat{\Psi}_{\mathrm{NS}}$ as

$$
\mu \rightarrow \frac{2}{\Delta\left(\frac{1}{2}-\rho\right)} \mu, \quad \tilde{\Psi}_{\mathrm{NS}}\left(k_{j}\right) \rightarrow \frac{1}{(-i \pi / 2) \Delta\left(\frac{1}{2}\left[1+\beta_{j}^{2}-k_{j}^{2}\right]\right)} \tilde{\Psi}_{\mathrm{NS}}\left(k_{j}\right)
$$

we get our main result

$$
\mathcal{A}\left(k_{1}, k_{2}, k_{3}\right)=\mu^{*} \text {. }
$$

Remarkably, this amplitude is of the same form as the bosonic one [10].

It is natural to expect that this feature continues to be true for $N$-point $(N \geq 4)$ functions. In fact, for $k_{1}, k_{2}, k_{3} \geq \alpha_{0}$, $k_{4} \leq \alpha_{0}<0$ (and without screening charges), the four-point function turns out to be

$$
\mathcal{A}\left(k_{1}, k_{2}, k_{3}, k_{4}\right)=(s+1) \mu^{s} \text {, }
$$

with the same redefinition of the cosmological constant and the primary superfields. In order to get the amplitude for general $k_{i}$, one may argue, as in Ref. [10], that nonanalyticity comes entirely from massless intermediate states and one 
may calculate the amplitude by using the analyticity of the one-particle irreducible (1PI) correlators. After setting $\mu=1$, we obtain the four-point function for all $k_{i}$ :

$$
\mathcal{A}\left(k_{1}, k_{2}, k_{3}, k_{4}\right)=-\alpha_{-}\left[\left|k_{1}+k_{2}-\alpha_{0}\right|+\left|k_{1}+k_{3}-\alpha_{0}\right|+\left|k_{1}+k_{4}-\alpha_{0}\right|\right]+\mathcal{A}_{1 \mathrm{PI}},
$$

with $\mathcal{A}_{\mathrm{IPI}}=-\frac{1}{2}\left(1+\alpha^{2}\right)$. Compare this with Eq. (37) in Ref. [10]. The analogy to the bosonic case is obvious. A detailed account will appear elsewhere.

The close connection to the bosonic amplitudes was suggested from super KP systems [20], and more recently, from supermatrix models [21]. We think, however, that our demonstration is more direct.

In conclusion, we calculated the three- and four-point functions of super Liouville theory coupled to a super Coulomb gas (without screening charges) on a sphere and found that they are essentially the same as those of the usual Liouville theory, obtained in Ref. [11]. As a by-product we get the supersymmetric generalization of formula (B.9) of Ref. [19] $(s=2 m+1)$,

$$
\begin{aligned}
& \frac{1}{s !} \int \prod_{i=1}^{s} d^{2} z_{i} d^{2} \tilde{\theta} \prod_{i=1}^{s}\left|z_{i}+\tilde{\theta} \theta_{i}\right|^{2 \alpha} \prod_{i=1}^{s}\left|1-z_{i}\right|^{2 \beta} \prod_{i<j}^{s}\left|\mathbf{z}_{i j}\right|^{4 \rho} \\
& =(-1)^{m} \pi^{2 m+1} \rho^{2 m} \Delta\left(\frac{1}{2}-\rho\right)^{2 m+1} \\
& \quad \times \prod_{i=1}^{m} \Delta(2 i \rho) \prod_{i=0}^{m} \Delta\left(\frac{1}{2}+(2 i+1) \rho\right) \prod_{i=0}^{m} \Delta(1+\alpha+2 i \rho) \Delta(1+\beta+2 i \rho) \Delta(-\alpha-\beta+(2 i-4 m) \rho) \\
& \quad \times \prod_{i=1}^{m} \Delta\left(\frac{1}{2}+\alpha+(2 i-1) \rho\right) \Delta\left(\frac{1}{2}+\beta+(2 i-1) \rho\right) \Delta\left(-\frac{1}{2}-\alpha-\beta+(2 i-4 m-1) \rho\right) .
\end{aligned}
$$

After completing this work, we found Refs. [17] and [22], which deal with the same issue, and, in fact, complement the present work.

K.H. would like to thank the members of Instituto de Física Teórica, UNESP, for their hospitality extended to him and FAPESP (Grant No. 90/1799-9) for the financial support. He also acknowledges communications on 2D gravity with $\mathrm{Y}$. Tanii and with $\mathrm{N}$. Ishibashi. D.D. thanks FAPESP (Grant No. 90/2246-3) for financial support. The work of E.A. and M.C.B.A. is partially supported by CNPq.

(a) Present address: Department of Physics, Kyushu University, Fukuoka 812, Japan.

[1] E. Brézin and V. A. Kazakov, Phys. Lett. B 236, 144 (1990); M. R. Douglas and S. H. Shenker, Nucl. Phys. B335, 635 (1990); D. J. Gross and A. A. Migdal, Phys. Rev. Lett. 64, 127 (1990).

[2] A. M. Polyakov, Phys. Lett. 103B, 207 (1981).

[3] T. L. Curtright and C. B. Thorn, Phys. Rev. Lett. 48, 1309 (1982); E. Braaten, T. L. Curtright, and C. B. Thorn, Phys. Lett. 118B, 115 (1982); Ann. Phys. (N.Y.) 147, 365 (1983); E. Braaten, T. L. Curtright, G. Gandour, and C. B. Thorn, Phys. Rev. Lett. 51, 19 (1983); Ann. Phys. (N.Y.) 153, 147 (1984); J. L. Gervais and A. Neveu, Nucl. Phys. B199, 50 (1982); B209, 125 (1982); B224, 329 (1983); B238, 123 (1984); B238, 396 (1984); E. D'Hoker and R. Jackiw, Phys. Rev. D 26, 3517 (1982); T. Yoneya, Phys. Lett. 148B, 111 (1984).

[4] F. David, Mod. Phys. Lett. A 3, 1651 (1988); J. Distler and H. Kawai, Nucl. Phys. B321, 509 (1989).

[5] N. Seiberg, in Proceedings of the 1990 Yukawa International Seminar on Common Trends in Mathematics and Quantum Field Theory, and Cargese Meeting on Random Surfaces, Quantum Gravity and Strings, 1990 (unpub- lished); J. Polchinski, Strings '90 Conference, College Station, Texas, 1990 [Nucl. Phys. B357, 241 (1991)].

[6] A. M. Polyakov, Phys. Lett. 103B, 211 (1981).

[7] J. F. Arvis, Nucl. Phys. B212, 151 (1983); B218, 309 (1983); O. Babelon, Nucl. Phys. B258, 680 (1985); Phys. Lett. 141B, 353 (1984); T. Curtright and G. Ghandour, Phys. Lett. 136B, 50 (1984).

[8] J. Distler, Z. Hlousek, and H. Kawai, Int. J. Mod. Phys. A 5, 391 (1990).

[9] M. Goulian and M. Li, Phys. Rev. Lett. 66, 2051 (1991).

[10] P. Di Francesco and D. Kutasov, Phys. Lett. B 261, 385 (1991).

[11] VI. S. Dotsenko, Mod. Phys. Lett. A 6, 3601 (1991).

[12] Y. Kitazawa, Phys. Lett. B 265, 262 (1991).

[13] N. Sakai and Y. Tanii, Prog. Theor. Phys. 86, 547 (1991).

[14] A. Gupta, S. P. Trivedi, and M. B. Wise, Nucl. Phys. B340, 475 (1990).

[15] See also M. Bershadsky and I. R. Klebanov, Phys. Rev. Lett. 65, 3088 (1990); Nucl. Phys. B360, 559 (1991); A. M. Polyakov, Mod. Phys. Lett. A 6, 635 (1991).

[16] M. A. Bershadsky, V. G. Knizhnik, and M. G. Teitelman, Phys. Lett. 151B, 131 (1985); D. Friedan, Z. Qiu, and S. Shenker, Phys. Lett. 151B, 37 (1985).

[17] P. Di Francesco and D. Kutasov, Report No. PUTP-1276 (unpublished).

[18] E. Martinec, Phys. Rev. D 28, 2604 (1983).

[19] VI. S. Dotsenko and V. Fateev, Nucl. Phys. B240, 312 (1984); B251, 691 (1985).

[20] P. Di Francesco, J. Distler, and D. Kutasov, Mod. Phys. Lett. A 5, 2135 (1990).

[21] L. Alvarez-Gaumé and J. L. Mañez, Mod. Phys. Lett. A 6. 2039 (1991).

[22] L. Alvarez-Gaumé and Ph. Zaugg, Phys. Lett. B 273, 81 (1991); K. Aoki and E. D'Hoker, Report No. UCLA/91/TEP/33, 1991 (unpublished). 